\title{
Universal Precaution in Otorhinolaryngology Surgery
}

\author{
Shrestha Bikash Lal* \\ Department of Otorhinolaryngology and Head and Neck Surgery, Kathmandu \\ University Hospital, Nepal
}

*Corresponding author: Bikash Lal Shrestha, Associate Professor, Department of

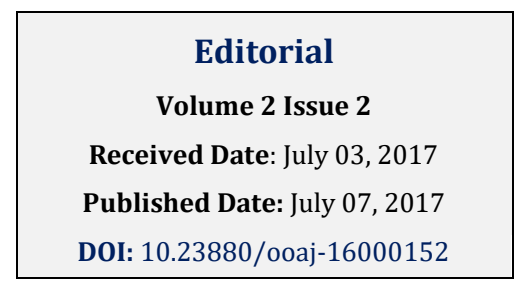

Otorhinolaryngology and head and neck surgery, Dhulikhel Hospital, Kathmandu University Hospital, Kavre, Nepal, Email: bikash001@hotmail.com

\section{Editorial}

The surgeons whether they are general surgeons, gynecologists or otorhinolaryngologists (ENT surgeons), are at risk from blood borne pathogens like hepatitis, HIV during interventions or surgeries. The developing countries health care workers are at particular risk because of the prevalence of such pathogens in under developed and developing countries. The risk of transmission is either through percutaneous injury or muco-cutaneous injuries. The transmission of infection is high with the percutaneous injury but the contact with skin and mucus membrane has equal possibility. The otolaryngologists usually take precaution to avoid needle stick injuries but few pay attention on blood or body fluid splashes into eye which is perhaps also major mode of transmission of infection. The otolaryngologists usually perform surgeries which have greater chance of blood or body fluid splashes into the eye like mastoid surgeries, head and neck surgeries, tonsillectomies, direct laryngoscopies among few of them to mention. They usually does not take any precaution to tackle the problem with the splashes and directly at risk of transmission of life threatening infections. Since the risk is very high with every surgeries, so it is mandatory to take the universal precaution while performing every bit of otolaryngology surgeries by the ENT surgeons. This will not only reduces the risk of life threatening infections but also assure the surgeons from protection and can even perform the surgeries without any stress. 